\title{
ADUBAÇÃO MINERAL DA CANDEIA (Eremanthus erythropappus (DC.) McLeish)
}

\author{
Nelson Venturin*, Patrícia Aparecida de Souza**, Renato Luiz Grisi de Macedo***, \\ Francisco Dias Nogueira**** \\ * Eng. Florestal, Dr., Depto. de Ciência Florestal, UFLA, Bolsista do CNPq - venturin@ufla.br \\ ** Eng. Florestal, Dr., Programa DCR/UFAM/CNPq/FAPEAM - patriciaapsouza@yahoo.com.br \\ *** Eng. Florestal, Eng. Agrônomo, Dr., Depto. Ciências Florestais, UFLA, Bolsista do CNPq - rgrisi@ufla.br \\ **** Eng. Agrônomo, Dr., EMBRAPA, EPAMIG - fdias@lavras.br
}

Recebido para publicação: 18/06/2004 - Aceito para publicação: 10/06/2005

\begin{abstract}
Resumo
Adubação mineral da candeia (Eremanthus erythropappus (DC.) McLeish). Com o objetivo de avaliar os aspectos nutricionais e os efeitos da omissão de nutrientes no desenvolvimento de plântulas de candeia (Eremanthus erythropappus (DC.) McLeish), foi conduzido um experimento em casa-devegetação. Como substrato foi utilizado um Latossolo Vermelho-Amarelo, de baixa fertilidade natural, coletado no município de Itumirim (MG), a uma profundidade de 20 a $40 \mathrm{~cm}$. Foram utilizados 10 tratamentos dispostos em delineamento de blocos inteiramente casualizados, com cinco repetições e uma planta por vaso, usando-se a técnica do nutriente faltante. Foram aplicados um tratamento completo com (N, P, K, Ca, Mg, S, B e Zn), outros oito com omissão de um nutriente por vez (-N, -P, $-\mathrm{K},-\mathrm{Ca},-\mathrm{Mg},-\mathrm{S},-\mathrm{B}$ e $-\mathrm{Zn}$ ) e um como testemunha (substrato natural). Foram avaliadas as seguintes característica aos 110 dias do plantio: altura da parte aérea, diâmetro de colo e produção de matéria seca da parte aérea e das raízes. Os resultados obtidos nas condições do presente trabalho permitem concluir que: a) Não houve crescimento das plantas de candeia nos tratamentos-testemunha e na ausência dos nutrientes $\mathrm{P}$ e $\mathrm{N}$; b) $\mathrm{O}$ nível de fertilidade do solo natural não permitiu o desenvolvimento das mudas de candeia; c) As limitações nutricionais da candeia em ordem decrescente são: $\mathrm{P}>\mathrm{N}>\mathrm{S}>\mathrm{Mg}=\mathrm{B}>\mathrm{Ca}>\mathrm{K}>\mathrm{Zn}$.

Palavras-chave: Espécie nativa; mudas; elemento faltante.
\end{abstract}

\begin{abstract}
Mineral fertilization of candeia (Eremanthus erythropappus (DC.) McLeish). This study evaluated the nutritional aspects and the effect of nutrient omission in development of candeia seedlings (Eremanthus erythropappus (DC.) McLeish). The experiment, based on missing-nutrient technique, was developed in a greenhouse, and used as substrate a low fertility oxisoil on 10 treatments, arranged in fully randomized block design, with 5 replicates and one plant per pot. It was applied a complete treatment with $(\mathrm{N}, \mathrm{P}, \mathrm{K}, \mathrm{Ca}, \mathrm{Mg}, \mathrm{S}, \mathrm{B}$, and $\mathrm{Zn})$, other eight elements with one missing element at a time $(-\mathrm{N},-\mathrm{P},-\mathrm{K},-\mathrm{Ca},-\mathrm{Mg},-\mathrm{S},-\mathrm{B}$, and $-\mathrm{Zn})$, and a control (natural substrate). The evaluation after 110 days measured the following variables: total height, ground level diameter, and dry matter. Based on the results allow the following conclusions: a) Candeia is strongly exigent in $\mathrm{P}$ and $\mathrm{N}$; b) The level of natural soil's fertility did not allow the development of candeia's seedlings; c) Candeia's nutritional limitation in decreasing order is $\mathrm{P}>\mathrm{N}>\mathrm{S}>\mathrm{Mg}=\mathrm{B}>\mathrm{Ca}>\mathrm{K}>\mathrm{Zn}$.

Keywords: Native species; seedlings; missing element.
\end{abstract}

\section{INTRODUÇÃO}

A espécie arbórea Eremanthus erythropappus (DC.) McLeish, popularmente conhecida como candeia, pertence à família Asteraceae, é nativa do Brasil e se adapta bem a solos pouco férteis e rasos. A sua principal utilização tem sido na produção de moirões com alta resistência ao apodrecimento, ao ataque de cupins e a bactérias do solo (Lorenzi, 1992). Recentemente, a candeia revelou-se como matéria-prima de grande qualidade para a produção de óleo essencial, cujo principal componente, o 
alfabisabolol, possui propriedades antiflogísticas, antibacterianas, antimicóticas, dermatológicas e espasmódicas (Scolforo et al., 2004).

A espécie é considerada por Lorenzi (1992) árvore de hábitat especial, pois em áreas nativas é encontrada em altitudes em torno de 1000 metros, nas chamadas "terras frias", onde o solo se classifica como Cambissolo e Litossolo, com afloramento de rochas. Durante o seu crescimento, é típica a formação de caules tortuosos, o que pode ser parcialmente dependente de sua nutrição mineral, sendo essa uma característica que às vezes dificulta seu uso.

A utilização de espécies nativas, como as candeias, entre outras, em plantios com fins comerciais torna-se comprometida pelo pouco conhecimento do comportamento silvicultural dessas espécies (Carpanezzi et al., 1976), principalmente em relação às suas exigências nutricionais. Visando suprir essa necessidade, vários trabalhos vêm sendo realizados utilizando-se a técnica do nutriente faltante.

A técnica do nutriente faltante é simples e segura para a identificação de deficiências nutricionais. Ela envolve o crescimento de plantas sob condições de campo ou de casa-de-vegetação, em que é testado um tratamento completo (com todos os nutrientes necessários em doses adequadas) e uma série de tratamentos incompletos, nos quais é feita a omissão de um nutriente de cada vez. De acordo com Braga (1983), para evitar que o resultado seja influenciado pela deficiência de outros nutrientes, nos tratamentos são adicionadas fontes dos demais nutrientes em quantidades não limitantes e nem tóxicas. O mesmo cuidado deve-se ter em relação à quantidade do elemento estudado. Sanchez e Salinas (1981) consideram como deficiência severa quando a produção de matéria seca cai a $40 \%$ a menos do que o tratamento completo.

Por meio da técnica do nutriente faltante, podem-se obter informações importantes, como a definição de quais nutrientes são deficientes, a importância relativa dessa deficiência, a velocidade de redução da fertilidade do solo (Chaminade, 1972) e a referência semiquantitativa da necessidade de adubação (Malavolta, 1980).

A produção de matéria seca total das plantas de freijó (Cordia goeldiana) foi afetada pelos tratamentos com omissão de N, P, Ca, K, S e Mg (Frazão, 1985) e da algaroba (Prosopis juliflora) pela ausência de N, K, P, Mg e Ca (Haag et al., 1986).

Carniel et al. (1993) observaram, em resposta à adubação em campo, que as espécies embaúba (Cecropia sp.), ipê-mirim (Stenolobium stans), fedegoso (Senna macranthera), canafístula (Senna multijulga) e faveira (Peltophorum dubium) tiveram o crescimento afetado pela falta de P, com exceção de ipê-mirim (Stenolobium stans). Entre elas, o fedegoso e a faveira mostraram as maiores restrições em crescimento quando da omissão de $\mathrm{N}$. Todas mostraram um baixo requerimento de $\mathrm{K}$ e $\mathrm{Mg}$ e elevada exigência nutricional em S. A ausência de Ca não afetou os teores foliares.

Duboc et al. (1996a) verificaram que os elementos N, P, Ca e S foram limitantes ao crescimento da copaíba (Copaifera langsdorffii) em solo com pequena disponibilidade desses nutrientes. As plantas mostraram pouca reação frente aos nutrientes $\mathrm{Mg}, \mathrm{K}, \mathrm{B}$ e $\mathrm{Zn}$, porque a disponibilidade original desses elementos no solo estudado era satisfatória. As omissões dos nutrientes $\mathrm{K}, \mathrm{Ca}$ e $\mathrm{Mg}$ afetaram a absorção de S pelas plantas. Para o jatobá (Hymenaea courbaril L.), os nutrientes mais limitantes foram N, P, Ca, $\mathrm{Mg}$, S e K. A disponibilidade de B e Zn no solo adubado com esses elementos foi superior às exigências das plantas, que apresentaram sintomas de toxidez ou desequilíbrio (Duboc et al., 1996b).

Venturin et al. (2000) avaliaram os aspectos nutricionais e os efeitos da ausência de nutrientes no desenvolvimento de plântulas de candiúva (Trema micrantha). Os autores concluíram que o $\mathrm{N}$ foi o nutriente mais limitante para o crescimento em altura, e o N, o P e o B para o diâmetro, sendo que o tratamento completo afetou o desenvolvimento da espécie devido à toxidez do nutriente zinco.

Este trabalho tem por objetivo avaliar a adubação mineral e o efeito da omissão de nutrientes sobre o crescimento de mudas de candeia (Eremanthus erythropappus (DC.) McLeish) em casa-devegetação, usando-se a técnica do nutriente faltante.

\section{MATERIAIS E MÉTODOS}

O trabalho foi conduzido em casa-de-vegetação no Departamento de Ciências Florestais da Universidade Federal de Lavras (UFLA). Como substrato, foi utilizado um Latossolo VermelhoAmarelo, de baixa fertilidade natural, coletado no município de Itumirim (MG), a uma profundidade de 20 a $40 \mathrm{~cm}$, para evitar a utilização da camada fértil do solo, que poderia mascarar o efeito dos 
fertilizantes.

$\mathrm{O}$ solo foi secado ao ar, passado em peneira de $5 \mathrm{~mm}$ e armazenado em sacos plásticos. Foram tomadas amostras para determinações físicas e químicas do material do solo antes e depois de se adicionar os nutrientes, e os resultados constam da Tabela 1. As análises físicas constaram de: determinação da textura (Método do Densímetro) e da densidade de partículas (Método do Balão Volumétrico), realizadas no Laboratório de Física do Solo da Universidade Federal de Lavras.

As análises químicas e físicas do solo foram feitas através dos seguintes métodos: $\mathrm{pH}\left(\mathrm{H}_{2} \mathrm{O}-\right.$ Relação 1:2,5); matéria orgânica (Método de Walkley e Black); $\mathrm{N}$ total (Método de Kjeldahl por destilação a vapor); $\mathrm{P} \mathrm{e} \mathrm{K}\left(\mathrm{HCl} 0,05 \mathrm{~mol}_{\mathrm{c}} \mathrm{L}^{-1}+\mathrm{H}_{2} \mathrm{SO}_{4} 0,025 \mathrm{~mol}_{\mathrm{c}} \mathrm{L}^{-1}\right)$, segundo Vettori (1969); $\mathrm{Ca}, \mathrm{Mg}$, $\mathrm{Al} \mathrm{e} \mathrm{H}+\mathrm{Al}$ (extrator $\mathrm{KCl} 1$ mol $\left._{\mathrm{c}} \mathrm{L}^{-1}\right)$; $\mathrm{Zn}, \mathrm{Cu}, \mathrm{Fe}$ e $\mathrm{Mn}\left(\mathrm{HCl} 0,05 \mathrm{~mol}_{\mathrm{c}} \mathrm{L}^{-1}+\mathrm{H}_{2} \mathrm{SO}_{4} 0,25 \mathrm{~mol}_{\mathrm{c}} \mathrm{L}^{-1}\right)$, segundo Viets Júnior e Lindsay (1973); $\mathrm{S}\left(\mathrm{Ca}\left(\mathrm{H}_{2} \mathrm{PO}_{4}\right)_{2} * \mathrm{H}_{2} \mathrm{O}+500 \mathrm{ppm} \mathrm{P}\right)$, conforme Tedesco et al. (1985); B (água quente), segundo descrição de Jackson (1970); e areia, silte e argila (Método do Densímetro). Essas análises foram realizadas no Laboratório de Fertilidade do Solo da Universidade Federal de Lavras (Tabela 1).

Tabela 1. Componentes químicos e físicos do solo ao natural e após a adubação com macro e micronutrientes.

Table 1. Chemical and physical components of the natural soil and after fertilization with macro and micronutrients.

\begin{tabular}{|c|c|c|}
\hline Parâmetros & Solo natural & Adubação completa \\
\hline $\mathrm{pH}(1)$ & 5,3 & 5,6 \\
\hline Mat. Org. $\left(\text { g.dag }^{-1}\right)^{(2)}$ & 0,3 & 0,4 \\
\hline $\mathrm{N}$ total $\left(\mathrm{mg} \cdot \mathrm{m}^{-3}\right)^{(3)}$ & 3,0 & 21,0 \\
\hline $\mathrm{P}\left(\mathrm{mg} \cdot \mathrm{dm}^{-3}\right)^{(4)}$ & 1,0 & 56,0 \\
\hline $\mathrm{K}\left(\mathrm{mg} \cdot \mathrm{dm}^{-3}\right)^{(4)}$ & 11,0 & 28,0 \\
\hline $\mathrm{S}\left(\mathrm{mg} \cdot \mathrm{dm}^{-3}\right)^{(5)}$ & 1,4 & 12,0 \\
\hline $\mathrm{Ca}\left(\mathrm{cmol}_{\mathrm{c}} \cdot \mathrm{dm}^{-3}\right)^{(6)}$ & 1,0 & 2,0 \\
\hline $\operatorname{Mg}\left(\mathrm{cmol}_{\mathrm{c}} \cdot \mathrm{dm}^{-3}\right)^{(6)}$ & 0,10 & 0,3 \\
\hline $\mathrm{Al}\left(\mathrm{cmol}_{\mathrm{c}} \cdot \mathrm{dm}^{-3}\right)^{(6)}$ & 0,10 & 1,8 \\
\hline $\mathrm{H}+\mathrm{Al}\left(\mathrm{cmol}_{\mathrm{c}} \cdot \mathrm{dm}^{-3}\right)^{(6)}$ & 1,30 & 1,6 \\
\hline $\mathrm{Zn}\left(\mathrm{mg} \cdot \mathrm{dm}^{-3}\right)^{(8)}$ & 1,35 & 3,0 \\
\hline $\mathrm{Cu}\left(\mathrm{mg} \cdot \mathrm{dm}^{-3}\right)^{(8)}$ & 20,8 & 28,0 \\
\hline $\mathrm{Fe}\left(\mathrm{mg} \cdot \mathrm{dm}^{-3}\right)^{(8)}$ & 11,3 & 15,0 \\
\hline $\operatorname{Mn}\left(\mathrm{mg} \cdot \mathrm{dm}^{-3}\right)^{(8)}$ & 1,4 & 1,6 \\
\hline $\mathrm{B}\left(\mathrm{mg} \cdot \mathrm{dm}^{-3}\right)^{(7)}$ & 0,2 & 0,6 \\
\hline Areia $\left(\text { g. dag }^{-1}\right)^{(9)}$ & 66,0 & 66,0 \\
\hline Silte $\left(\text { g.dag }^{-1}\right)^{(9)}$ & 10,0 & 10,0 \\
\hline Argila $\left(\text { g.dag }^{-1}\right)^{(9)}$ & 24,0 & 24,0 \\
\hline
\end{tabular}

As sementes de candeia foram coletadas em árvores selecionadas no campo e colocadas a germinar em um substrato composto por uma mistura de um terço de solo esterilizado, um terço de casca de arroz carbonizada e um terço de areia. Ao emitirem quatro pares de folhas, foram transplantadas duas mudas por vaso, com capacidade de 4 litros de solo. Após 30 dias foi feito o desbaste, deixando-se apenas uma planta por vaso.

A umidade dos vasos foi mantida, com água deionizada, a $60 \%$ da capacidade de campo e aferida diariamente, como proposto por Freire et al. (1980). Os vasos tiveram os fundos tampados, para evitar a perda de nutrientes.

Foram testados dez tratamentos, dispostos em um delineamento em blocos inteiramente 
casualizados, com cinco repetições, um vaso por repetição e uma planta por vaso. Os tratamentos foram os seguintes: T (Testemunha, substrato natural), C (Completo - contendo todos os nutrientes, que são: N, $\mathrm{P}, \mathrm{K}, \mathrm{Ca}, \mathrm{Mg}, \mathrm{S}, \mathrm{Zn}$ e B), -N (Completo menos nitrogênio), -P (Completo menos fósforo), -K (Completo menos potássio), -Ca (Completo menos cálcio), -Mg (Completo menos magnésio), -S (Completo menos enxofre), -Zn (Completo menos zinco) e -B (Completo menos boro).

A adubação completa consistiu em: $\mathrm{N}=150 \mathrm{mg} / \mathrm{kg}$ de solo, $\mathrm{P}=200 \mathrm{mg} / \mathrm{kg}, \mathrm{K}=150 \mathrm{mg} / \mathrm{kg}, \mathrm{Ca}$ $=75 \mathrm{mg} / \mathrm{kg}, \mathrm{Mg}=15 \mathrm{mg} / \mathrm{kg}, \mathrm{S}=50 \mathrm{mg} / \mathrm{kg}, \mathrm{B}=0,5 \mathrm{mg} / \mathrm{kg}, \mathrm{Cu}=1,5 \mathrm{mg} / \mathrm{kg}, \mathrm{Zn}=1,4 \mathrm{mg} / \mathrm{kg}, \mathrm{Mn}=20$ $\mathrm{mg} / \mathrm{kg}$ e $\mathrm{Fe}=25 \mathrm{mg} / \mathrm{kg}$. O N foi parcelado, aplicando-se $1 / 3$ no plantio (repicagem) e o restante em doses iguais aos 20, 50 e 80 dias. $\mathrm{O} \mathrm{K}$ foi aplicado aos 20 dias após a repicagem, em dose única.

Aos 110 dias após o plantio, foram avaliados o diâmetro do colo, a altura da parte aérea e a produção de matéria seca da parte aérea e das raízes. O diâmetro foi tomado no coleto, e a altura, do solo até ao ápice da planta. Após medidos os diâmetros e as alturas, as mudas foram cortadas rente ao solo e pesadas. O sistema radicular foi separado do solo, lavado e pesado. Ambas, raízes e parte aérea, foram secadas em estufa com circulação de ar a $70{ }^{\circ} \mathrm{C}$, até obtenção de peso constante. Na matéria seca da parte aérea foram determinadas também as concentrações de macro e micronutrientes, segundo Sarruge e Haag (1974). As análises foram feitas no Laboratório de Nutrição Mineral de Plantas da Universidade Federal de Lavras (UFLA).

Os dados obtidos foram submetidos à análise de variância e ao teste de médias segundo Scott e Knott (1974).

\section{RESULTADOS E DISCUSSÃO}

\section{Crescimento das plantas}

Os resultados referentes ao crescimento das plântulas de candeia são apresentados na Tabela 2.

Os tratamentos com omissão de $\mathrm{P}$ e a Testemunha não apresentaram produção de matéria verde, indicando que a baixa disponibilidade de $\mathrm{P}$ ou a baixa fertilidade do solo (Testemunha) não foram suficientes para as plantas manterem seu crescimento. Esses tratamentos foram retirados do experimento 60 dias após o plantio. Resultados semelhantes com a omissão do nutriente $\mathrm{P}$ foram obtidos com outras espécies florestais, como: cedro (Cedrela fissilis) (Silva e Muniz, 1995), peroba-rosa (Aspidosperma polyneuron) (Muniz e Silva, 1995), acácia-mangium (Acacia mangium), quaresmeira (Tibouchina granulosa), Aspidosperma polyneurom (Braga et al., 1995), pau-ferro (Caesalpinea ferrea), cássia amarela (Senna multijuga), pau-jacaré (Piptadenia gonoacantha) (Renó, 1994) e jacarandá-da-baía (Dalbergia nigra) (Venturin et al., 1997. No tratamento completo, as mudas de candeia apresentaram altura e diâmetro iguais ou superiores aos demais tratamentos. A omissão de N mostrou-se limitante, afetando em geral o crescimento das plantas em altura e diâmetro. Para o crescimento em altura, a ausência dos nutrientes $\mathrm{P}, \mathrm{K}, \mathrm{Ca}, \mathrm{Mg}, \mathrm{S}, \mathrm{Zn}$ e B mostraram-se indiferentes em relação ao tratamento completo. A falta de $\mathrm{N}$ e $\mathrm{S}$ afetou o crescimento em diâmetro do caule da candeia. Simões e Couto (1973) encontraram para o pinheiro-do-paraná resultados semelhantes, sendo que a omissão de $\mathrm{N}$ trouxe um forte desequilíbrio nutricional, inibindo o crescimento em altura e diâmetro.

$\mathrm{O}$ crescimento da parte aérea e radicular da candeia quanto à matéria seca foi reduzido pela ausência de N (Figura 1), e a relação raiz/parte aérea foi mais afetada na supressão de $\mathrm{Mg}$, embora estatisticamente não tenha havido diferença com a omissão de K, Ca e B (Tabela 2). Em relação ao sistema radicular, a falta de Zn proporcionou maior produção de matéria seca em 15\%, não diferindo estatisticamente do tratamento Completo. Resultado semelhante foi encontrado por Duboc et al. (1996b), estudando as exigências nutricionais do jatobá, que verificaram também uma baixa exigência de $\mathrm{Zn}$ para a espécie.

De maneira geral, o desenvolvimento das plantas de candeia foi afetado pela ausência de nutrientes em quase todos os tratamentos, com exceção do tratamento sem $\mathrm{Zn}$, no qual a produção de matéria seca da parte aérea e da matéria seca do sistema radicular foi maior do que a do tratamento completo, embora estatisticamente iguais (Figura 1), mostrando que possivelmente a candeia não seja exigente para esse nutriente. Isso é muito comum no caso dos micronutrientes. Normalmente, nessa fase de desenvolvimento, as quantidades presentes no solo, sob condições ideais em casa-de-vegetação, favorecem sua absorção e aproveitamento, mesmo estando abaixo de $1 \mathrm{mg} \cdot \mathrm{dm}^{-3}$ (Duboc et al., 1996a). 
Tabela 2. Altura, diâmetro, produção de matéria seca da parte aérea (MSPA) e do sistema radicular (MSSR) e relação raiz/parte aérea (R/PA) de mudas Eremanthus erythropappus (DC.) McLeish em função da omissão de nutrientes.

Table 2. Height, diameter, dry matter production of shoot (DMPS) and root (DMPR), and rate root/shoot (R/S) of Eremanthus erythropappus (DC.) McLeish seedlings in lacking nutrient.

\begin{tabular}{lccccc}
\hline Tratamento & Altura (cm) & Diâmetro (mm) & MSPA (g) & MSSR (g) & R/PA \\
\hline Testemunha & nd & nd & nd & nd & nd \\
Completo & $16,00 \mathrm{a}$ & $0,51 \mathrm{a}$ & $5,56 \mathrm{a}$ & $2,31 \mathrm{a}$ & $0,42 \mathrm{a}$ \\
$-\mathrm{N}$ & $8,40 \mathrm{~b}$ & $0,29 \mathrm{~b}$ & $1,05 \mathrm{c}$ & $0,50 \mathrm{~b}$ & $0,47 \mathrm{a}$ \\
$-\mathrm{P}$ & $\mathrm{nd}$ & nd & nd & nd & nd \\
$-\mathrm{K}$ & $15,66 \mathrm{a}$ & $0,43 \mathrm{a}$ & $5,58 \mathrm{a}$ & $1,99 \mathrm{a}$ & $0,35 \mathrm{~b}$ \\
$-\mathrm{Ca}$ & $16,60 \mathrm{a}$ & $0,46 \mathrm{a}$ & $5,29 \mathrm{a}$ & $1,70 \mathrm{a}$ & $0,33 \mathrm{~b}$ \\
$-\mathrm{Mg}$ & $12,62 \mathrm{a}$ & $0,40 \mathrm{a}$ & $3,94 \mathrm{~b}$ & $0,81 \mathrm{~b}$ & $0,23 \mathrm{~b}$ \\
$-\mathrm{S}$ & $12,90 \mathrm{a}$ & $0,33 \mathrm{~b}$ & $2,94 \mathrm{~b}$ & $1,36 \mathrm{~b}$ & $0,46 \mathrm{a}$ \\
$-\mathrm{B}$ & $15,60 \mathrm{a}$ & $0,43 \mathrm{a}$ & $3,52 \mathrm{~b}$ & $1,21 \mathrm{~b}$ & $0,35 \mathrm{~b}$ \\
$-\mathrm{Zn}$ & $17,10 \mathrm{a}$ & $0,45 \mathrm{a}$ & $5,75 \mathrm{a}$ & $2,66 \mathrm{a}$ & $0,46 \mathrm{a}$ \\
\hline Média & 14,36 & 0,41 & 4,20 & 1,56 & 0,38 \\
\hline CV (\%) & 20,85 & 16,84 & 26,22 & 34,22 & 23,45 \\
\hline
\end{tabular}

Letras distintas na coluna diferem entre si pelo teste de Scott e Knott ao nível de 5\% de probabilidade. MSPA = Matéria seca da parte aérea; MSSR = Matéria seca do sistema radicular; $\mathrm{R} / \mathrm{A}=$ Relação raiz/parte aérea; $\mathrm{g}$ = grama por vaso; $\mathrm{nd}=$ não determinado.

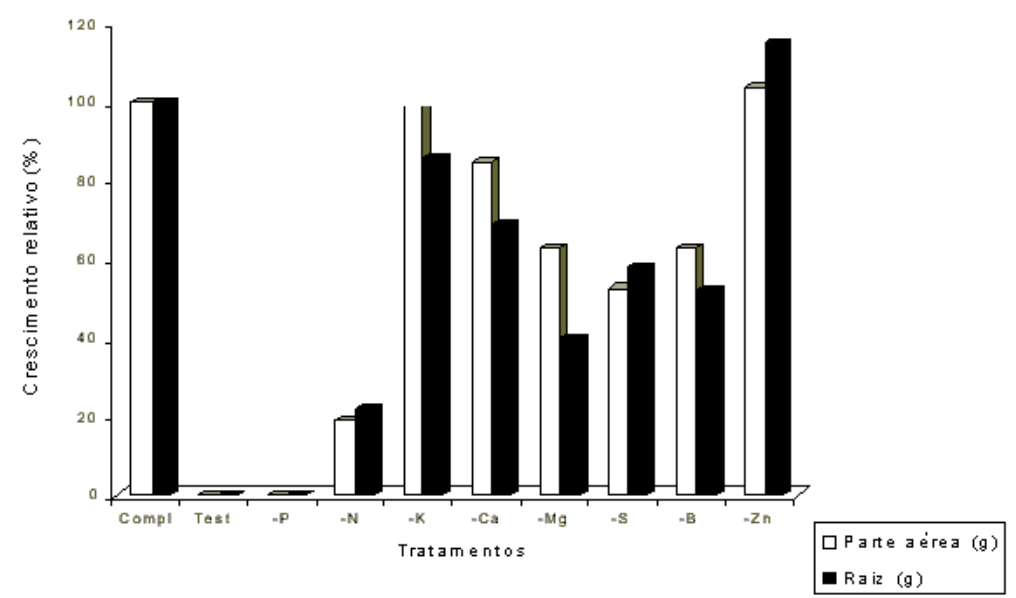

Figura 1. Crescimento relativo da parte aérea e raiz de mudas Eremanthus erythropappus (DC.) McLeish. Figure 1. Relative growth of shoot and root of Eremanthus erythropappus (DC.) McLeish seedlings.

Em termos de alteração na partição de matéria seca, entre raízes e parte aérea (relação R/PA), diante das mudanças de disponibilidade dos nutrientes no solo, observou-se que a candeia teve maior relação no tratamento completo e nas omissões de $\mathrm{N}, \mathrm{S}$ e $\mathrm{Zn}$ (Tabela 2), os quais não diferiram estatisticamente entre si. As ausências de $\mathrm{K}, \mathrm{Ca}, \mathrm{Mg}$ e B provocaram menor relação raiz/parte aérea. Esses nutrientes são importantes na ativação de enzimas do solo, sendo que a transformação do fósforo orgânico do solo (aproximadamente $60 \%$ do total) requer a presença de $\mathrm{K}$ e Mg na ativação da enzima fosfatase ácida (TPases), enquanto o Ca é importante constituinte da parede celular. O boro atua na dominância das gemas apicais e crescimento das plantas. 
A relação R/PA é comumente maior em ambiente de baixa fertilidade, podendo ser considerada uma estratégia da planta para retirar o máximo de nutrientes naquela condição (Clarkson, 1985). Portanto, a candeia apresenta estratégias distintas de comportamento quanto ao solo de baixa fertilidade, uma vez que a ausência de $\mathrm{Mg}$ foi a que mais reduziu o crescimento das raízes, porque a relação $\mathrm{K} / \mathrm{Ca}+\mathrm{Mg}$, não tendo sido otimizada, não permitiu que o crescimento se processasse na sua plenitude.

A seqüência de exigência nutricional apresentada pelas mudas de candeia em relação ao tratamento completo, considerando a produção de matéria seca da parte aérea em ordem decrescente, foi: $\mathrm{P}>\mathrm{N}>\mathrm{S}>\mathrm{Mg}=\mathrm{B}>\mathrm{Ca}>\mathrm{K}>\mathrm{Zn}$.

Os resultados ressaltam a importância de estudos dessa natureza, em que se busca caracterizar as exigências nutricionais de espécies florestais, as quais evoluíram e se adaptaram, ao longo de gerações, a determinadas condições pedoclimáticas e, portanto, podem apresentar características distintas de comportamento.

\section{Nutrição mineral das plantas}

Os teores dos nutrientes na matéria seca da parte aérea (MSPA) das plântulas de candeia estão apresentados na Tabela 3. Pode-se observar que os teores mais baixos de macro e micronutrientes encontram-se associados aos tratamentos sob omissão de cada respectivo nutriente.

$\mathrm{O}$ teor de $\mathrm{N}$ na parte aérea foi favorecido pela omissão de $\mathrm{S}$ (Tabela 3). Isso ocorreu porque houve efeito de concentração, pois os tratamentos que apresentaram os maiores teores de N na MSPA foram os de menor produção de matéria seca (Tabela 2). Como o enxofre é indispensável na síntese de proteínas, admite-se que o $\mathrm{K}$ ou mesmo a relação $\mathrm{K} / \mathrm{Ca}+\mathrm{Mg}$ não tenha favorecido a rota metabólica adequadamente e, portanto, tenha provocado menos produção de matéria seca.

Tabela 3. Teores de nutrientes na matéria seca da parte aérea de mudas Eremanthus erythropappus (DC.) McLeish.

Table 3. Concentration of nutrients in shoot dry matter of Eremanthus erythropappus (DC.) McLeish seedlings.

\begin{tabular}{|c|c|c|c|c|c|c|c|c|}
\hline \multirow{3}{*}{ Tratamento } & \multicolumn{8}{|c|}{ Teor de nutrientes } \\
\hline & $\mathbf{N}$ & $\mathbf{P}$ & $\mathbf{K}$ & $\mathbf{C a}$ & Mg & $\mathbf{S}$ & B & Zn \\
\hline & \multicolumn{6}{|c|}{ g.kg ${ }^{-1}$} & \multicolumn{2}{|c|}{ Mg.kg ${ }^{-1}$} \\
\hline Testemunha & nd & nd & nd & nd & nd & nd & nd & nd \\
\hline Completo & $1,79 \mathrm{c}$ & $0,70 b$ & $0,80 \mathrm{~b}$ & $9,27 b$ & $2,48 \mathrm{~b}$ & $0,97 \mathrm{c}$ & $14,58 \mathrm{c}$ & $244,23 c$ \\
\hline$-\mathrm{N}$ & $1,89 \mathrm{c}$ & $0,95 \mathrm{a}$ & $1,09 a$ & $8,13 b$ & $3,42 \mathrm{a}$ & $2,93 a$ & $20,86 a$ & $381,34 b$ \\
\hline$-\mathrm{P}$ & nd & nd & nd & nd & nd & nd & nd & nd \\
\hline$-\mathrm{K}$ & $2,54 \mathrm{c}$ & $0,56 b$ & $0,23 \mathrm{c}$ & $8,46 b$ & $3,62 \mathrm{a}$ & $0,96 \mathrm{c}$ & $17,72 b$ & $367,32 b$ \\
\hline$-\mathrm{Ca}$ & $2,12 \mathrm{c}$ & $1,22 \mathrm{a}$ & $0,97 \mathrm{a}$ & $4,56 \mathrm{c}$ & $3,11 \mathrm{~b}$ & $1,04 \mathrm{c}$ & $12,32 d$ & $550,68 \mathrm{a}$ \\
\hline$-\mathrm{Mg}$ & $3,00 \mathrm{~b}$ & $0,70 b$ & $0,93 b$ & 11,24 & $2,30 \mathrm{~b}$ & $1,12 \mathrm{c}$ & $13,39 d$ & $438,81 \mathrm{~b}$ \\
\hline$-\mathrm{S}$ & $3,80 \mathrm{a}$ & $0,88 b$ & $1,11 \mathrm{a}$ & $8,81 \mathrm{~b}$ & $2,89 \mathrm{~b}$ & $0,39 d$ & $15,62 \mathrm{c}$ & $284,88 \mathrm{c}$ \\
\hline$-B$ & $2,28 \mathrm{c}$ & $0,71 b$ & $1,06 \mathrm{a}$ & $8,21 b$ & $3,58 \mathrm{a}$ & $1,73 b$ & $5,97 \mathrm{e}$ & $360,16 b$ \\
\hline$-Z n$ & $2,45 \mathrm{c}$ & $1,17 \mathrm{a}$ & 0,790 & $8,86 b$ & $2,73 \mathrm{~b}$ & $0,98 \mathrm{c}$ & $16,68 \mathrm{~b}$ & $44,64 d$ \\
\hline Média & 2,49 & 0,83 & 0,88 & 8,45 & 3,02 & 1,27 & 14,64 & 334,01 \\
\hline CV (\%) & 21,78 & 26,2 & 15,1 & 12,1 & 35,3 & 16,3 & 11,20 & 19,58 \\
\hline
\end{tabular}

As letras distintas nas colunas diferem entre si pelo teste de Scott \& Knott a 5\%. nd = não determinado.

Os tratamentos com omissão de N, Ca e Zn apresentaram os maiores teores de P na MSPA (Tabela 3). Na falta de $\mathrm{Zn}$, possivelmente devido à não-ocorrência do mecanismo de inibição não competitiva, foi favorecida a absorção de P (Malavolta, 1980). O teor de P na ausência de Zn foi inferior $\left(1,17 \mathrm{~g} / \mathrm{kg}^{-1}\right.$, Tabela 3) ao encontrado por Braga et al. (1995) em Acacia mangium e quaresmeira (Tibouchina granulosa), com os teores de P de $1,4 \mathrm{~g} / \mathrm{kg}$ e 1,7 g/kg, respectivamente.

$\mathrm{O}$ teor de $\mathrm{K}$ na MSPA das plântulas de candeia, no tratamento com a omissão deste macronutriente, foi inferior ao teor do tratamento Completo (Tabela 3). Porém, as plantas apresentaram produção de matéria seca da parte aérea semelhante à do tratamento Completo, o que sugere que a 
espécie tenha mecanismos eficientes para crescer com pequenas quantidades do nutriente $\mathrm{K}$ no solo. Dias et al., (1991), para Acacia mangium, encontraram que o crescimento dessa espécie foi reduzido com a aplicação de K no substrato.

O menor teor de Ca ocorreu no tratamento com a omissão desse componente, e o mais elevado foi encontrado com a ausência de $\mathrm{Mg}$. $\mathrm{O}$ maior teor de Ca no tratamento sem $\mathrm{Mg}$ pode estar relacionado com a redução da inibição competitiva (Malavolta et al., 1997) sobre a absorção de Ca. Resultados semelhantes foram encontrados para o pinheiro-do-paraná por Simões e Couto (1973), que constataram que o tratamento sem $\mathrm{Mg}$ apresentou teor de Ca elevado de 9,4 g. $\mathrm{kg}^{-1}$.

$\mathrm{O}$ teor de $\mathrm{Mg}$ foi favorecido pelas ausências de $\mathrm{K}$ e $\mathrm{Ca}$, possivelmente devido à redução do antagonismo entre estes e o Mg (Malavolta et al., 1997). O teor de Mg encontrado para candeia de 2,48 g.kg ${ }^{-1}$ no tratamento Completo é inferior ao obtido por Braga et al. (1995) na MSPA de plantas de peroba-rosa, de 4,0 g.kg ${ }^{-1}$.

As plantas de candeia apresentaram teor de $\mathrm{S}$ inferior sob sua omissão (Tabela 3), sendo 2,5 vezes menor que o do tratamento Completo. Resultado semelhante foi encontrado por Duboc et al. (1996b) para o jatobá, que foi 2,6 vezes menor que o do tratamento Completo aplicado para essa espécie. $\mathrm{O}$ maior teor de $\mathrm{S}$ foi encontrado pela omissão de $\mathrm{N}$ (Tabela 3), na qual a competição aniônica foi reduzida, pois no tratamento Completo e nos demais, as fontes de $\mathrm{N}$ aplicadas foram na forma de nitrato. É ainda possível afirmar que a matéria seca foi reduzida com o aumento da concentração de S.

O maior teor de B na matéria seca da parte aérea foi encontrado no tratamento com ausência de N (Tabela 3), ocorrendo possivelmente devido ao efeito de concentração, já que a produção de MSPA deste tratamento foi menor (Tabela 2), e o menor teor foi encontrado quando se omitiu a sua aplicação.

$\mathrm{O}$ menor teor de $\mathrm{Zn}$ na MSPA de plantas de candeia foi encontrado pela omissão deste micronutriente, sendo inferior ao das outras omissões (Tabela 3). Considerando que a produção de matéria seca na ausência de Zn não diferiu da do tratamento Completo, pode-se inferir que a candeia tem mecanismos rizosféricos (exsudação de compostos orgânicos) que são solubilizados de compostos de zinco pouco disponíveis. Na ausência deste, houve aumento da altura de plantas e redução do diâmetro, sem alterar a produção de matéria seca e a relação R/PA (Tabela 2). Esses fatos indicaram que a disponibilidade de $\mathrm{Zn}$ no solo foi suficiente para o desenvolvimento das plantas, considerando a soma do teor contido no solo (Tabela1) com o teor contido no tratamento completo. O teor de $\mathrm{Zn}$ encontrado no solo original e após a adubação neste experimento foi respectivamente $1,35 \mathrm{mg} / \mathrm{dm}^{3}$ e $3 \mathrm{mg} / \mathrm{dm}^{3}$, muito acima do intervalo crítico proposto por Lopes e Carvalho (1988), de 0,8 a $1,0 \mathrm{mg} / \mathrm{dm}^{3}$. O teor crítico interno de Zn proposto por Couto et al. (1985) para Eucalyptus grandis é inferior a $11 \mathrm{mg} / \mathrm{kg}$, e teores superiores a $200 \mathrm{mg} / \mathrm{kg}$ proporcionam limitações ao crescimento.

\section{CONCLUSÕES}

Nas condições do presente estudo, pode-se concluir que:

- o nível de fertilidade do solo natural estudado não permitiu o desenvolvimento das plantas de candeia;

- a ausência de $\mathrm{P}$ e $\mathrm{N}$ afetou drasticamente o crescimento das plântulas;

- Os teores de $\mathrm{K}, \mathrm{Ca}, \mathrm{S}, \mathrm{B}$ e $\mathrm{Zn}$ na matéria seca da parte aérea de candeia foi reduzido nas omissões destes nutrientes; e

- as limitações nutricionais para a candeia, em ordem decrescente, são: $\mathrm{P}>\mathrm{N}>\mathrm{S}>\mathrm{Mg}=\mathrm{B}>\mathrm{C}>$ $\mathrm{K}>\mathrm{Zn}$.

\section{REFERÊNCIAS}

BRAGA, F. A.; VALE, F. R.; VENTURIN, N. Exigências nutricionais de quatro espécies florestais. Revista Árvore, MG, v.19, n.1, p.18-32, 1995.

BRAGA, J. M. Avaliação da fertilidade do solo: ensaios de campo. Viçosa: UFV, 1983. 101 p. 
CARNIEL, T.; LIMA, H. N.; VALE, F. R. do, Resposta à adubação no campo de cinco espécies arbóreas nativas do sudeste brasileiro. In: CONGRESSO BRASILEIRO DE CIÊNCIA DO SOLO, 24, 1993, Goiânia. Resumos..., Goiânia: SBCS, 1993. p.209-210.

CARPANEZZI, A. A.; BRITO, J. O.; FERNANDES, P. Teor de macro e micronutrientes em folhas de diferentes idades de algumas essências florestais nativas. Anais da E.S.A. "Luiz de Queiroz", Piracicaba, v.23, p.225-232, 1976.

CHAMINADE, R. Recherches sur fertilite et la fertilisation des sols em régions tropicales. L'Agronomie Tropicales, Paris, v.27, n.9, p.891-904, sept, 1972.

CLARKSON, D. T. Adaptações morfológicas e fisiológicas das plantas a ambientes de baixa fertilidade. In: SIMPÓSIO SOBRE RECICLAGEM DE NUTRIENTES E AGRICULTURA DE BAIXOS INSUMOS NOS TRÓPICOS, 1984, Ilhéus. Anais... Ilhéus: CEPLAC/SBCS, 1985. p.45-75.

COUTO, C.; NOVAIS, R. F. de; BARROS, N. F. de. Resposta do eucalipto à aplicação de zinco em amostras de solos de cerrado, Revista Árvore, Viçosa, MG, v.9, n.2, p.134-148, 1985.

DIAS, L. E; ALVAREZ V. V. H.; BRIENZA JUNIOR, S. Formação de mudas de Acacia mangium Will: 2 Resposta a Nitrogênio e Potássio. Revista Árvore, Viçosa, MG, v.15, n.1, p.11-22, 1991.

DUBOC, E.; VENTURIN, N.; VALE, F. R. do. Fertilização de plântulas de Copaifera langsdorffii Desf. (Óleo copaíba). Cerne, Lavras, v.2, n.2, p.31-47, 1996a.

DUBOC, E.; VENTURIN, N.; VALE, F. R. do. Nutrição do jatobá (Hymenala courbaril L. var. Stilbocarba (Haene) Lee et lang). Cerne, Lavras, v.2, n.1, p.138-152, 1996 b.

FRAZÃO, D. A. C. Sintomatologia das carências de macronutrientes em casa de vegetação e recrutamento de nutrientes pelo freijó (Cordia goeldiana Huber) aos 2, 3, 4 e 8 anos de idade implantado em latossolo amarelo distrófico, Belterra, Pará. 1985. Piracicaba, 194f. Dissertação (Doutorado em Agronomia) Escola Superior de Agricultura "Luiz de Queiroz".

FREIRE, J. C.; RIBEIRO, M. A. V.; BAHIA, G. V. Resposta do milho cultivado em casa de vegetação a níveis de água em solo da região de Lavras, MG. Revista Brasileira de Ciência do Solo, Campinas, v.4, n.1, p.518, 1980 .

HAAG, H. P; MEDEIROS, A. A. de; FRANÇA, A. F. de S. Desnutrição de macronutrientes em plantas de algaroba. IPEF, Piracicaba, n.32, p.53-55, 1986.

JACKSON, M. L. Analises quimicas de suelos. 2. ed. Barcelona: [s.n.] 1970. 662 p.

LOPES, A. S.; CARVALHO, J. G. Micronutrientes: critérios de diagnose para solo e planta, correção de deficiências e excessos. In: BORKERT, C. M.; LANTMANN, A. F. (Eds.). Enxofre e micronutrients na agrigultura brasileira. Londrina: EMBRAPA - CNPSo/IAPAR/SBCS, 1988. p. 133-78.

LORENZI, H. Árvores brasileiras: manual de identificação e cultivo de plantas arbóreas nativas do Brasil. Nova Odessa: Plantarum, 1992. 352p.

MAlavolta, E.; VITTI, G. C.; OLIVEIRA, S. A. Avaliação do estado nutricional das plantas: princípios e aplicações. 2.ed. Piracicaba: POTAFOS, 1997. 319p.

MALAVOLTA. E. Elementos de nutrição mineral de plantas. São Paulo: Ceres, 1980. 220 p.

MUNIZ, A. S.; SILVA, M. A. G. da. Exigências nutricionais de mudas de peroba-rosa (Aspidosperma polyneuron Muller Arg.) em solução nutritiva. Revista Árvore, Viçosa, MG, v.19, n.2, p.263-271, 1995.

RENÓ, N. B. Requerimentos nutricionais e resposta ao fósforo e fungo micorrízico de espécies arbóreas nativas do sudeste brasileiro. Lavras, 1994. 62f. Dissertação (Mestrado em Solos e Nutrição de Plantas). Escola Superior de Agricultura "Luiz de Queiroz".

SANCHES, P. A.; SALINAS, J. G. Low-input technology for managing oxisols and ultisols in Tropical America. Advances in Agronomy, San Diego, Califórnia, v.34, p.279-406, 1981.

SARRUGE, J. R.; HAAG, H. P. Análises químicas em plantas. Piracicaba: ESALQ, 1974. 56p. 
SCOLFORO, J. R. S; OLIVEIRA, A. D.; DAVIDE, A. C. Manejo sustentado das candeias Eremanthus erytropappus (DC.) McLeish e Eremathus incanus (Less.) Less. Disponível em: <WWW.nucleoestudo.ufla.br/nemaf/candeia $>$ Acesso em: 05 mai. 2004.

SCOTT, A. J.; KNOTT, M. A cluster analysis method for grouping means in the analysis of variance. Biometrics, Washington, DC, v.30, p. 507-512, 1974.

SILVA, M. A. G.; MUNIZ, A. S. Exigências nutricionais de cedro (Cedrella fissilis Velloso) em solução nutritiva. Revista Árvore, Viçosa, MG, v.19, n.3, p.415-425, 1995.

SIMÕES, J. W.; COUTO, H. T. Z. do. Efeitos da omissão na alimentação do pinheiro do Paraná Araucaria angustifolia (Bert.) O. Ktze cultivado em vaso. IPEF, Piracicaba, v.4, n.7, p.3-40, out. 1973.

TEDESCO, M. J.; VOLKWEISS, S. J.; BOHNEN, H. Análise do solo, plantas e outros materiais. Porto Alegre: UFRGS, 1985. (Boletim Técnico, 5).

VENTURIN, N.; SILVA, E. B.; MORATO, M. I. R. Avaliação das exigências nutricionais do jacarandáda-bahia (Dalbergia nigra). In: CONGRESSO BRASILEIRO DE CIÊNCIA DO SOLO, 26., 1997, Rio de Janeiro. Anais... Rio de Janeiro: SBCS/EMBRAPA/CNPS, 1997. 1 CDROOM.

VENTURIN, N.; SOUZA, P. A. de; VENTURIN, R. P. Avaliação nutricional da candiúva (Trema micrantha L. Blumes) em casa de vegetação. Floresta, Rio de Janeiro, v.29 (1/2), p.15-26, Nov. 2000.

VETTORI, L. Métodos de análise de solo. Rio de Janeiro: [s.n.] 1969. 34p. (Boletim Téenico 7).

VIETS JUNIOR, F. G.; LINDSAY, W. L. Testing soils for zinc, cooper, manganese and iron. In: Soil testing and plant analysis. Madison: Soil Science society of America, 1973 . p. 329-488. 